chlorides to the metallic form. Hartwich had first done this in the 1880 s with small rusty samples at $500{ }^{\circ} \mathrm{C}$ in a hydrogen atmosphere; the process is widely used in industry, but not in museums.

A cylinder of depth $126 \mathrm{~cm}$, radius $23 \mathrm{~cm}$ was loaded with the samples (for instance six cannon balls) which were then placed in a furnace in hydrogen. Reduction was carried out at 600 to $800{ }^{\circ} \mathrm{C}$ and after a period of about a day the process was complete-not only were oxides absent but chlorides too had been reduced to iron. Chemical analyses show that there was generally no trace of chlorine and all the iron was in the metallic form. Subsequent coating with an antirust agent and accelerated rusting tests show that the objects are now fully protected against corrosion. They now sit in the Wasa Museum-uncorroding and with the right shape.

\section{Success of super labs at Cologne meeting}

\section{from a Correspondent}

THE Spring meeting of the Cologne Institute of Genetics (February 22-24) opened with a symposium on tumour viruses which, as W. Doerfler (Cologne Institute of Genetics)-who organised it-pointed out, really deaît with DNA viruses, since even the RNA viruses discussed by $P$. Vogt (University of Southern California) and J. Dahlberg (University of Wisconsin) have a transitional DNA phase.

Transcriptional maps of the adenovirus genome in productive infection are emerging both from the Wallenberg Laboratory at Uppsala and from Cold Spring Harbor. L. Philipson (Wallenberg Laboratory) described the techniques used by his group to obtain adenovirus DNA fragments from cleavage with restriction enzyme and labelled either in the heavy or the light chain. The amount of labelled DNA could be measured after the templates had been hybridised with excess unlabelled RNA, to give the quantity of DNA transcribed from either the light or the heavy strand of the restriction enzyme fragments.

Philipson's results proved entirely compatible with those of the Cold Spring Harbor group, presented at the symposium by W. Keller. The highlights of Keller's talk were the description of a new approach to sequencing viral DNA using a series of restriction enzyme cleavages which should greatly facilitate what is normally a long and painful process, and the results of the analyses of six hamster cell lines transformed with adenoviruses.

All the transformed lines seem to hand, at least a portion of restriction fragment $F$ located near the middle of the DNA towards the $\mathrm{C}$ fragment end was absent from all the lines. Keller speculated that the adenovirus DNA was integrated into the host chromosomes and that the functions contained in the retained portion of fragment $A$ were necessary for its main'tenance.

A. Lewis (National Institutes of Health), in a thorough analysis of the non-defective adenovirus-SV40 hybrids, described the unequivocal mapping by heteroduplex analyses of the order of several early SV40 functions. The question whether viral DNA becomes integrated in'io 'transformed' lymphocytes was raised by $T$. Lindahl (Karolinska Institute), who showed that the link between Epstein-Barr virus DNA to lymphoblast DNA was not stable in alkali, although a substantial portion of the viral DNA banded in caesium chloride with host DNA. Only when the DNA was sheared into small fragments were the host and viral DNA sequences effectively separated by isopycnic banding.

Vogt effectively summarised the work on RNA tumour viruses with a lucid exposition of the dilemmas with which analyses of the siructure of oncornavirus are fraught. Most of the data, including those on the kinetic complexity of the genome, suggest that it is haploid and segmented. But reservations on this conclusion musi remain because all of the RNA segments, and not just one, decrease in size on the mutation of sarcoma viruses to become defective or leukaemogenic.

The symposium concluded with a tour de force: the announcement of the sequence of the transfer RNA molecule which serves as the primer for DNA synthesised on the 70S RNA template by reverse transcriptase. This was the fruit of a collaborative project involving Dahlberg at Madison (who presented the paper) and M. Bishop's laboratory at the University of California in San Francisco, and it illustrated one of the most conspicuous incidental points brought out by the meeting. This was the contrast in the accomplishments of the large institutionalised collaborative groups such as those at Uppsala, Cold Spring Harbor, NIH, and on the San Francisco-Madison axis, and those of the smaller laboratories, both European and American, represented at the meeting. It was the superlabs which, by approaching complex problems from several directions simultaneously, emerged as the more effective, despite the inevitable loss of scientific identity resulting from the dilution of credit among individual contributions.

have a portion of the terminal restriction fragment $\mathrm{A}$ and all but one contain at least a portion of the $\mathrm{C}$ fragment located at the other end. On the other

\section{Perfusing the female reproductive system}

from our

\section{Steroid Biochemistry Correspondent}

WITH the well known limitations of most in vitro techniques for studying the metabolism of tissues, the use of perfusion or superfusion techniques has become more popular. In the field of reproductive biology, interesting results have been obtained by perfusion of the human ovary, placenta or foetoplacental unit. Tojo et al. (Amer. J. Obstet. Gynec., 118, 119-129; 1974) have now achieved in vitro perfusion of the human uterus and fallopian tubes as well as the ovary.

Before removing the utero-tubalovarian unit, the arterial pressure, pulse rate and blood flow were measured and the pressure wave form of the uterine arteries was recorded. The unit was connected to the perfusing machine through the bilateral uterine arteries and the bilateral uterine and ovarian veins and was perfused in such a way that the pulse rate and pressure of the input of the perfusate were similar to those expected in the uterine artery in vivo. This was achieved by using a Belloframtype artificial heart driven by a liquid amplifier, a pressure wave form regulator for the perfusate, an oxygenator, organ chamber, suction pump for venous return and an autoregulatory thermocontrol mechanism.

The viability of the unit during perfusion was tested by measuring the erythrocyte count, haematocrit, $p \mathrm{O}_{2}$, $p \mathrm{CO}_{2}$ and $p \mathrm{H}$ values and the haemoglobin, lactate and pyruvate content of the perfusate.

Tojo et al. obtained successful perfusions of three utero-tubal-ovarian units, one obtained during the menstrual period and two during the mid-luteal phase of women with normal ovarian function. Addition of human chorionic gonadotrophin (HCG) to the perfusate increased the progesterone secretion from the ovary obtained during the luteal phase but not from that obtained during menstruation. When tissues obtained at the thirteenth week of pregnancy were perfused, there was a rapid increase in the HCG content and a gradual increase in the content of human placental lactogen (HPL) of the perfusate. Active nucleic acid synthesis was shown to occur in the trophoblastic tissue.

These preliminary results suggest that the utero-tubal-ovarian unit maintains its viability for some considerable time when perfused. If this is confirmed in further investigations, application of this method should help in the elucidation of many problems of reproductory physiology and pathology. 